\title{
Impurity-induced moments in underdoped cuprates
}

\author{
G. Khaliullin \\ Max-Planck-Institut für Physik komplexer Systeme, Nöthnitzer Str. 38, D-01187 Dresden, Germany \\ and Kazan Physicotechnical Institute of the Russian Academy of Sciences, 420029 Kazan, Russia \\ R. Kilian \\ Max-Planck-Institut für Physik komplexer Systeme, Nöthnitzer Str. 38, D-01187 Dresden, Germany \\ S. Krivenko \\ Kazan Physicotechnical Institute of the Russian Academy of Sciences, 420029 Kazan, Russia \\ P. Fulde \\ Max-Planck-Institut für Physik komplexer Systeme, Nöthnitzer Str. 38, D-01187 Dresden, Germany
}

(02 July 1997)

\begin{abstract}
We examine the effect of a nonmagnetic impurity in a two-dimensional spin liquid in the spingap phase, employing a drone-fermion representation of spin- $1 / 2$ operators. The properties of the local moment induced in the vicinity of the impurity are investigated and an expression for the nuclear-magnetic-resonance Knight shift is derived, which we compare with experimental results. Introducing a second impurity into the spin liquid an antiferromagnetic interaction between the moments is found when the two impurities are located on different sublattices. The presence of many impurities leads to a screening of this interaction as is shown by means of a coherent-potential approximation. Further, the Kondo screening of an impurity-induced local spin by charge carriers is discussed.
\end{abstract}

74.25.Ha, 74.72.-h, 75.20.Hr, 76.60.-k

\section{INTRODUCTION}

Substitution of $\mathrm{Cu}$ ions in the conduction planes of high- $T_{c}$ cuprates by different nonmagnetic ions presents an important experimental tool for the study of the metallic state of these strongly correlated systems. Unusual effects have been revealed especially when these materials were doped with $\mathrm{Zn}$, Al, or Ga. Among these interesting features is the appearance of a magnetic moment derived from the observation of a CurieWeiss behavior of the magnetic susceptibility. Further studies have been performed by electron paramagnetif resonance 3 (EPR) and nuclear magnetic resonance 3 (NMR) experiments. Measurements of the NMR Knight shift indicate that the impurity-induced local moments reside predominantly on $\mathrm{Cu}$ sites neighboring the dopant. 10 It has been argued that the appearance of these localmoments can account for a significant reduction of $T_{c}$.

On the theoretical side, impurity-induced moments have been studied for a variety of quantum disordered spin systems, includine for instance, spin ladders and spin-Peierls systems, cuprates 10 A common feature of these systems is the existence of a (pseudo-) gap in the spin-excitation spectrum. This spin gap is related to the singlet-pairing correlations in the ground state, which can be described in terms of resonance valence bonds 11 (RVB) or a valencebond solid (VBS) state.12 Within this picture the appearance of a Curie-type susceptibility can be understood as an unpaired spin that is generated by the substitution of a spin site by a vacancy.

In the present paper we investigate the effect of nonmagnetic impurities on the local magnetic properties of weakly doped cuprates. In order to describe the spin degrees of freedom of these systems we start with the Heisenberg antiferromagnet on a square lattice, employing a drone-fermion representation of spin$1 / 2$ operators. 13 A mean-field decoupling of the Hamiltonian leads to solytions corresponding to a RVB state in the flux phase 14.15 that exhibits a pseudogap in the spin-excitation spectrum. A single spin vacancy introduced into the spin liquid produces a local moment which is predominantly located on the sites close to the vacancy. We derive an expression for the impurity-induced NMR Knight shift and fit it to experimental data. Introducing a second vacancy into the spin liquid leads to an antiferromagnetic interaction between the two moments. In the presence of many impurities this interaction is screened as is shown by using a coherent-potential approximation (CPA). We further analyze the effect of nonmagnetic impurities on the relaxation of the nuclear magnetic moment in terms of local spin fluctuations induced by the vacancies. Finally, we discuss qualitatively the low-temperature Kondo screening of the impurityinduced spins by charge carriers.

\section{THE MODEL}

We start from the Hamiltonian of a two-dimensional square lattice spin-1/2 Heisenberg antiferromagnet 


$$
H=J \sum_{\langle i j\rangle} \boldsymbol{S}_{i} \cdot \boldsymbol{S}_{j}
$$

where \langle\rangle indicates summation over pairs of nearestneighbor sites. The spin operators in the Hamiltonian (11) are commonly expressed in terms of pseudo-fermions which requires us to impose the restriction that each site is occupied by exactly one pseudoparticle. Since it is rather difficult to account for these local constraints one usually treats them onlyon the average. Here we will follow a different approach 16 by employing a drone-fermion description for spin- $1 / 2$ operators given by 13

$$
S_{i}^{+}=f_{i}^{\dagger} \chi_{i}, \quad S_{i}^{-}=\chi_{i} f_{i}, \quad S_{i}^{z}=f_{i}^{\dagger} f_{i}-\frac{1}{2},
$$

where $S_{i}^{ \pm}=\left(S_{i}^{x} \pm i S_{i}^{y}\right) / \sqrt{2}$. The operator $f_{i}^{\dagger}$ creates a spinless fermion at site $i$; the presence (absence) of an $f$ fermion corresponds to an up (down) spin state. The real drone-fermion operator $\chi_{i}=\chi_{i}^{\dagger}$ with commutation rules $\left[\chi_{i}, \chi_{j}\right]_{+}=\delta_{i j}$ is needed to provide the proper commutation rules for spin operators on different sites. Expressing the Hamiltonian (11) in terms of the representation (2) one obtains

$$
\begin{aligned}
H= & J \sum_{\langle i j\rangle}\left\{\left(f_{i}^{\dagger} \chi_{i} \chi_{j} f_{j}+\text { H.c. }\right)\right. \\
& \left.+\left(f_{i}^{\dagger} f_{i}-\frac{1}{2}\right)\left(f_{j}^{\dagger} f_{j}-\frac{1}{2}\right)\right\} .
\end{aligned}
$$

Mean-field solutions to the Hamiltonian (3) corresponding to the RVB states of conventional fermion mean-field theory are found by employing a Hartree-Fock factorization with bond parameters $i \Delta_{i j}=\left\langle\chi_{j} \chi_{i}\right\rangle$ and $i \tilde{\Delta}_{i j}=\left\langle f_{j}^{\dagger} f_{i}\right\rangle$ defined for nearest-neighbor pairs, yielding the following mean-field Hamiltonian: 16

$$
\begin{aligned}
H_{\mathrm{MF}}= & J \sum_{\langle i j\rangle}\left\{\left(-i\left(\tilde{\Delta}_{i j}+\Delta_{i j}\right) f_{i}^{\dagger} f_{j}\right.\right. \\
& \left.\left.-i \tilde{\Delta}_{i j} \chi_{i} \chi_{j}+\text { H.c. }\right)+2 \tilde{\Delta}_{i j} \Delta_{i j}+\tilde{\Delta}_{i j}^{2}\right\} .
\end{aligned}
$$

For spin rotational symmetry of the ground state $\tilde{\Delta}_{i j}=$ $\Delta_{i j}$ holds. In the following we assume the order parameter to depend only on relative site indices: $\Delta_{i j}=\Delta_{\delta}$ with $\boldsymbol{\delta} \in\{\hat{\boldsymbol{x}},-\hat{\boldsymbol{x}}, \hat{\boldsymbol{y}},-\hat{\boldsymbol{y}}\}$, where $\hat{\boldsymbol{x}}$ and $\hat{\boldsymbol{y}}$ are the lattice unit vectors.

Dividing the square lattice into two sublattices $A$ and $B$, we define $i \in A$ and $j \in B$. The mean-field Hamiltonian (4) can then be diagonalized by expressing the $f$ and $\chi$ operators in momentum representation and employing the canonical transformation

$$
\begin{aligned}
f_{\boldsymbol{k}}^{A} & =\left(a_{\boldsymbol{k}}+b_{\boldsymbol{k}}\right) / \sqrt{2}, \\
f_{\boldsymbol{k}}^{B} & =\left(a_{\boldsymbol{k}}-b_{\boldsymbol{k}}\right) \exp \left(i \varphi_{\boldsymbol{k}}\right) / \sqrt{2}, \\
\chi_{\boldsymbol{k}}^{A} & =\left(c_{\boldsymbol{k}}+d_{\boldsymbol{k}}\right) / \sqrt{2}, \\
\chi_{\boldsymbol{k}}^{B} & =\left(c_{\boldsymbol{k}}-d_{\boldsymbol{k}}\right) \exp \left(i \varphi_{\boldsymbol{k}}\right) / \sqrt{2},
\end{aligned}
$$

where $\exp \left(i \varphi_{\boldsymbol{k}}\right)=\Delta_{\boldsymbol{k}}^{*} /\left|\Delta_{\boldsymbol{k}}\right|$ with the definition $\Delta_{\boldsymbol{k}}=$ $i \sum_{\delta} \Delta_{\delta} \exp (-i \boldsymbol{k} \boldsymbol{\delta})$. Thus, the Hamiltonian (4) becomes

$$
\begin{aligned}
H_{\mathrm{MF}}= & \sum_{\boldsymbol{k} \in \mathrm{MBZ}} \xi_{\boldsymbol{k}}\left(-a_{\boldsymbol{k}}^{\dagger} a_{\boldsymbol{k}}+b_{\boldsymbol{k}}^{\dagger} b_{\boldsymbol{k}}\right) \\
& +\sum_{\boldsymbol{k} \in \mathrm{MBZ} / 2} \xi_{\boldsymbol{k}}\left(-c_{\boldsymbol{k}}^{\dagger} c_{\boldsymbol{k}}+d_{\boldsymbol{k}}^{\dagger} d_{\boldsymbol{k}}\right)
\end{aligned}
$$

with $\xi_{\boldsymbol{k}}=2 J\left|\Delta_{\boldsymbol{k}}\right|$. The $k$ summations extend over the full and half magnetic Brillouin zone, respectively, and the constant terms in Eq. (1) have been omitted.

The fermionic spectrum $E(\boldsymbol{k})= \pm \xi_{\boldsymbol{k}}$ is determined by the symmetry exhibited by the bond parameter $\Delta_{\delta}$. In a phase of mixed symmetry defined by

$$
\Delta:=\Delta_{\hat{x}}=-\Delta_{-\hat{x}}=\Delta_{\hat{y}}=\Delta_{-\hat{y}}
$$

one finds

$$
\xi_{\boldsymbol{k}}=\frac{D}{\sqrt{2}} \sqrt{\sin ^{2} k_{x}+\cos ^{2} k_{y}},
$$

where $D=4 \sqrt{2} J \Delta$ is the half-width of the band and the lattice constant has been set equal to unity. At low energies the density of states is determined by the neighborhood of the two isolated roots of Eq. (9) and exhibits a V-shaped pseudogap $\rho^{0}(\omega)=2|\omega| /\left(\pi D^{2}\right)$ corresponding to the one found in the phase of conventional fermion mean-field theories. 14.15

The bandwidth parameter $D$ in Eq. (9) is obtained from the self-consistency relation

$$
D=\frac{2 \sqrt{2} J}{N} \sum_{k} \eta_{k} \tanh \left(\frac{D \eta_{k}}{2 \sqrt{2} T}\right)
$$

where $\eta_{\boldsymbol{k}}=\left(\sin ^{2} k_{x}+\cos ^{2} k_{y}\right)^{1 / 2}, N$ is the number of sites, and $k_{B}=1$. In the limit of $T \rightarrow 0$ Eq. (10) reduces to

$$
D_{0}=\frac{2 \sqrt{2} J}{N} \sum_{k} \eta_{k}
$$

yielding a numerical value of $D_{0} \approx 1.355 \mathrm{~J}$. The correction $\delta D(T)$ to $D_{0}$ for small but finite temperatures is given by

$$
\delta D(T)=-\frac{32 J}{\pi}\left(\frac{T}{D_{0}}\right)^{3} .
$$

In the following we study the effect of a spin vacancy located at site 0 (sublattice $A$ ) on the local magnetic properties of the system described by Hamiltonian (7). We simulate this spin defect by decoupling site 0 from the rest of the system. This is done in two steps: First, the drone-fermion bond parameter connecting the impurity site with its nearest neighbors is set to zero, $i \Delta_{0, \delta}=\left\langle\chi_{\boldsymbol{\delta}} \chi_{0}\right\rangle=0$. This decouples the drone fermions 
of site 0 from the rest of the system. Then, a local chemical potential $\lambda_{0} \rightarrow \infty$ acting on site 0 is added to Hamiltonian (7),

$$
H_{\mathrm{imp}}=H_{\mathrm{MF}}+\lambda_{0} f_{0}^{\dagger} f_{0} .
$$

It induces an empty site at $\boldsymbol{R}=0$ with respect to the spinon $f$ operators and suppresses the low-energy $f$-fermion degrees of freedom at this site. We will show below that the presence of the local potential also leads to a vanishing spinon bond parameter connecting the impurity site with its nearest neighbors, $i \tilde{\Delta}_{0, \delta}=\left\langle f_{\delta}^{\dagger} f_{0}\right\rangle=0$, and therefore completely decouples the impurity. Further, one infers $\tilde{\Delta}_{\boldsymbol{R}, \boldsymbol{R}+\boldsymbol{\delta}}=\Delta_{\boldsymbol{R}, \boldsymbol{R}+\boldsymbol{\delta}}$ for all $\boldsymbol{R} \in A$ which manifests the equivalent treatment of $f$ and drone fermions as required by spin rotational symmetry.

\section{LOCAL MOMENT}

In this section we study the uniform properties and the spatial distribution of a local moment induced by a spin vacancy. We first calculate the impurity contributions to the spinon density of states and the uniform spin susceptibility by means of the uniform Green's function $G\left(i \omega_{n}\right)=-1 / N \sum_{\boldsymbol{r}}\left\langle T_{\tau} f_{\boldsymbol{r}}(\tau) f_{\boldsymbol{r}}^{\dagger}(0)\right\rangle_{\omega_{n}}$ given by

$$
G\left(i \omega_{n}\right)=N G^{0}\left(i \omega_{n}\right)+\delta G\left(i \omega_{n}\right)
$$

with fermionic frequencies $\omega_{n}=(2 n+1) \pi T$. The Green's function in the absence of the impurity $G^{0}\left(i \omega_{n}\right)$ and the correction $\delta G\left(i \omega_{n}\right)$ due to the impurity are given by

$$
\begin{aligned}
G^{0}\left(i \omega_{n}\right) & =\frac{1}{N} \sum_{\boldsymbol{k}, \nu} g_{\nu}^{0}\left(i \omega_{n} ; \boldsymbol{k}\right), \\
\delta G\left(i \omega_{n}\right) & =\frac{1}{N} \sum_{\boldsymbol{k}, \nu} g_{\nu}^{0}\left(i \omega_{n} ; \boldsymbol{k}\right) T\left(i \omega_{n}\right) g_{\nu}^{0}\left(i \omega_{n} ; \boldsymbol{k}\right)
\end{aligned}
$$

with $g_{\nu}^{0}\left(i \omega_{n} ; \boldsymbol{k}\right)=1 /\left(i \omega_{n}-(-1)^{\nu} \xi_{\boldsymbol{k}}\right), \nu \in\{1,2\}$. The $T$ matrix in Eq. (15) describes successive scattering at the vacancy and is obtained from usual scattering theory; in the limit $\lambda_{0} \rightarrow \infty$ it is $T\left(i \omega_{n}\right)=-1 / G^{0}\left(i \omega_{n}\right)$, yielding

$$
\delta G\left(i \omega_{n}\right)=\frac{\partial}{\partial i \omega_{n}} \ln G^{0}\left(i \omega_{n}\right)
$$

Evaluating Eq. (16) one obtains

$$
\delta G\left(i \omega_{n}\right)=\frac{1}{i \omega_{n}}\left(1-\ln ^{-1} \frac{D}{\left|\omega_{n}\right|}\right)
$$

From Eq. (17) we calculate the impurity correction to the density of states $\delta \rho(\omega)=-1 / \pi \operatorname{Im} \delta G\left(\omega+i 0^{+}\right)$:

$$
\delta \rho(\omega)=\delta(\omega)-\frac{1}{2|\omega|} \frac{1}{(\pi / 2)^{2}+\ln ^{2}(D /|\omega|)} .
$$

The first and second terms on the right-hand side of Eq. (18) describe, respectively, the formation of a spinon bound state at the Fermi surface and the destruction of a spinon singlet in the spin background. Integrating Eq. (18) over all occupied states one finds a reduction in the number of particles by $1 / 2$. This is in agreement with the fact that the local chemical potential $\lambda_{0}$ induces an empty site at $\boldsymbol{R}=0$ with respect to the $f$ fermions, while in the absence of $\lambda_{0}$ the average $f$-fermion occupation number is $1 / 2$.

With the help of Eq. (17) we evaluate the impurity contribution $\delta \chi(T)$ to the uniform spin susceptibility by employing the relation

$$
\delta \chi(T)=T \sum_{i \omega_{n}} \frac{\partial}{\partial i \omega_{n}} \delta G\left(i \omega_{n}\right) .
$$

For $T \ll J$ one obtains

$$
\delta \chi(T)=\frac{1}{4 T}\left(1-\ln ^{-1} \frac{D}{T}\right) .
$$

The formation of a spinon bound state is thus found to lead to a Curie-like spin susceptibility of a free spin $1 / 2$ with logarithmic correction We note that conventional fermion mean-field theory 10 only reproduces one half of the above result. This shortcoming has its origin in the mean-field treatment of the local constraints on the pseudoparticle occupation number which is characteristic for those theories.

We analyze the spatial distribution of the impurityinduced moment by means of the local Green's function $G\left(i \omega_{n} ; \boldsymbol{R}, \boldsymbol{R}\right)=-\left\langle T_{\tau} f_{\boldsymbol{R}}(\tau) f_{\boldsymbol{R}}^{\dagger}(0)\right\rangle_{\omega_{n}}$ which is given by

$$
G\left(i \omega_{n} ; \boldsymbol{R}, \boldsymbol{R}\right)=G^{0}\left(i \omega_{n}\right)+\delta G\left(i \omega_{n} ; \boldsymbol{R}, \boldsymbol{R}\right) .
$$

Depending upon whether $\boldsymbol{R}$ lies on the $A$ or $B$ sublattice the impurity contribution $\delta G\left(i \omega_{n} ; \boldsymbol{R}, \boldsymbol{R}\right)$ is

$$
\begin{aligned}
& \delta G\left(i \omega_{n}, \boldsymbol{R}, \boldsymbol{R}\right)=\left|A\left(i \omega_{n} ; \boldsymbol{R}\right)\right|^{2} / G^{0}\left(i \omega_{n}\right), \quad \boldsymbol{R} \in A, \\
& \delta G\left(i \omega_{n}, \boldsymbol{R}, \boldsymbol{R}\right)=-\left|B\left(i \omega_{n} ; \boldsymbol{R}\right)\right|^{2} / G^{0}\left(i \omega_{n}\right), \quad \boldsymbol{R} \in B
\end{aligned}
$$

with

$$
\begin{aligned}
A\left(i \omega_{n} ; \boldsymbol{R} \in A\right)= & \frac{1}{N} \sum_{\boldsymbol{k}, \nu} g_{\nu}^{0}\left(i \omega_{n} ; \boldsymbol{k}\right) \exp (i \boldsymbol{k R}), \\
B\left(i \omega_{n} ; \boldsymbol{R} \in B\right)= & \frac{1}{N} \sum_{\boldsymbol{k}, \nu} g_{\nu}^{0}\left(i \omega_{n} ; \boldsymbol{k}\right)(-1)^{\nu+1} \\
& \times \exp \left(-i \varphi_{\boldsymbol{k}}+i \boldsymbol{k} \boldsymbol{R}\right) .
\end{aligned}
$$

Evaluating Eqs. (22) one finds

$$
\begin{aligned}
\delta G\left(i \omega_{n} ; \boldsymbol{R}, \boldsymbol{R}\right)= & \frac{4 \Phi_{A}(\boldsymbol{R})}{\pi D^{2}} K_{0}^{2}\left(\frac{\sqrt{2} R\left|\omega_{n}\right|}{D}\right) \\
& \times i \omega_{n} \ln ^{-1} \frac{D}{\left|\omega_{n}\right|} \quad \text { for } \boldsymbol{R} \in A, \\
\delta G\left(i \omega_{n} ; \boldsymbol{R}, \boldsymbol{R}\right)= & -\frac{4 \Phi_{B}(\boldsymbol{R})}{\pi D^{2}} K_{1}^{2}\left(\frac{\sqrt{2} R\left|\omega_{n}\right|}{D}\right) \\
& \times i \omega_{n} \ln ^{-1} \frac{D}{\left|\omega_{n}\right|} \quad \text { for } \boldsymbol{R} \in B,
\end{aligned}
$$


where the $K_{i}$ are MacDonald's functions 17 and the angular dependence is determined by

$$
\begin{aligned}
\Phi_{A}(\boldsymbol{R} \in A) & =\left(1+\cos \pi R_{x}\right) / 2, \\
\Phi_{B}(\boldsymbol{R} \in B) & =\left(1-\left(2 R_{x}^{2} / R^{2}-1\right) \cos \pi R_{x}\right) / 2 .
\end{aligned}
$$

From Eqs. (24) we determine the impurity contribution to the local density of states, $\delta \rho(\omega ; \boldsymbol{R})=-1 / \pi \operatorname{Im} \delta G(\omega+$ $\left.i 0^{+} ; \boldsymbol{R}, \boldsymbol{R}\right)$. It is found that the local moment resides predominantly on the $B$ sublattice; for $R|\omega| \ll J$ one obtains

$$
\begin{aligned}
\delta \rho(\omega ; \boldsymbol{R} \in B)= & \frac{2 \Phi_{B}(\boldsymbol{R})}{\pi R^{2}}\left(\frac{\delta(\omega)}{\ln (D /|\omega|)}+\frac{1}{2|\omega|}\right. \\
& \left.\times \frac{1}{(\pi / 2)^{2}+\ln ^{2}(D /|\omega|)}\right),
\end{aligned}
$$

while $\delta \rho(\omega ; \boldsymbol{R} \in A)$ is negligible.

Our particular interest is the local spin polarization in the vicinity of the impurity as this quantity is needed below to derive an expression for the NMR Knight shift which we will compare to experimental data. In the presence of a uniform magnetic field the spin polarization on site $\boldsymbol{R}$ can be obtained from the local spin susceptibility

$$
\delta \chi(T ; \boldsymbol{R})=T \sum_{i \omega_{n}} \frac{\partial}{\partial i \omega_{n}} \delta G\left(i \omega_{n} ; \boldsymbol{R}, \boldsymbol{R}\right) .
$$

Evaluating Eq. (27) for $R|\omega| \ll J$ with $\boldsymbol{R} \in B$ one obtains

$$
\delta \chi(T ; \boldsymbol{R} \in B)=\frac{1}{2 \pi T} \frac{\Phi_{B}(\boldsymbol{R})}{R^{2}} f(T, R) \ln ^{-1} \frac{D}{T}
$$

with a correction factor

$$
f(T, R)=\left(x K_{1}(x)\right)^{2}, \quad x=\frac{4 \sqrt{2} T R}{\pi D},
$$

which approaches unity for $T R \ll J$. Due to the $R^{-2}$ decay of $\delta \chi(T ; \boldsymbol{R})$ the spin polarizability is found to be strongest on the nearest-neighbor sites of the vacancy. Contributions from sublattice $A$ are again negligible. The logarithmic correction in Eq. (28) is due to the marginal character of the bound state: its spectral weight at a given lattice site vanishes logarithmically as $\omega \rightarrow 0$. Integrating Eq. (28) over $\boldsymbol{R}$ one again obtains the contribution to the uniform susceptibility $\delta \chi(T)=1 /(4 T)$ as required.

We now turn to the spinon bond parameter $i \tilde{\Delta}_{\boldsymbol{R}, \boldsymbol{R}+\boldsymbol{\delta}}=$ $\left\langle f_{\boldsymbol{R}+\delta}^{\dagger} f_{\boldsymbol{R}}\right\rangle$ defined for $\boldsymbol{R} \in A$, which we express in terms of the Green's function $G\left(i \omega_{n} ; \boldsymbol{R}, \boldsymbol{R}+\boldsymbol{\delta}\right)=$ $-\left\langle T_{\tau} f_{\boldsymbol{R}}(\tau) f_{\boldsymbol{R}+\boldsymbol{\delta}}^{\dagger}(0)\right\rangle_{\omega_{n}}$ :

$$
\tilde{\Delta}_{\boldsymbol{R}, \boldsymbol{R}+\boldsymbol{\delta}}=-i T \sum_{i \omega_{n}} G\left(i \omega_{n} ; \boldsymbol{R}, \boldsymbol{R}+\boldsymbol{\delta}\right) .
$$

Splitting the Green's function into an unperturbed and an impurity-correction term one obtains

$$
\tilde{\Delta}_{\boldsymbol{R}, \boldsymbol{R}+\boldsymbol{\delta}}=\tilde{\Delta}_{\delta}+\delta \tilde{\Delta}_{\boldsymbol{R}, \boldsymbol{R}+\boldsymbol{\delta}}
$$

with

$$
\begin{gathered}
\tilde{\Delta}_{\delta}=-i T \sum_{i \omega_{n}} G^{0}\left(i \omega_{n} ; 0, \boldsymbol{\delta}\right), \\
\delta \tilde{\Delta}_{\boldsymbol{R}, \boldsymbol{R}+\boldsymbol{\delta}}=-i T \sum_{i \omega_{n}} \delta G\left(i \omega_{n} ; \boldsymbol{R}, \boldsymbol{R}+\boldsymbol{\delta}\right),
\end{gathered}
$$

where

$$
\begin{aligned}
G^{0}\left(i \omega_{n} ; 0, \boldsymbol{\delta}\right)= & B\left(i \omega_{n} ; \boldsymbol{\delta}\right), \\
\delta G\left(i \omega_{n} ; \boldsymbol{R}, \boldsymbol{R}+\boldsymbol{\delta}\right)= & A^{*}\left(i \omega_{n} ; \boldsymbol{R}\right) \\
& \times B\left(i \omega_{n} ; \boldsymbol{R}+\boldsymbol{\delta}\right) / G^{0}\left(i \omega_{n}\right) .
\end{aligned}
$$

For $\boldsymbol{R}=0$ one finds $G^{0}\left(i \omega_{n} ; 0, \boldsymbol{\delta}\right)=-\delta G\left(i \omega_{n} ; 0, \boldsymbol{\delta}\right)$ which reconfirms the vanishing spinon bond parameter connecting the impurity site with its nearest neighbors, i.e., $\tilde{\Delta}_{0, \delta}=0$.

\section{KNIGHT SHIFT}

Experimentally, the spatial distribution of an impurity induced moment can be investigated by means of the NMR Knight shift $K(T)$ on either the impurity, $\mathrm{Cu}$, or in the case of $\mathrm{YBa}_{2} \mathrm{Cu}_{3} \mathrm{O}_{7}$ on the $\mathrm{Y}$ nuclei. Further information is obtained from the width of the NMR lines and the relaxation rates of the nuclear magnetic moment.

The Hamiltonian describing a nuclear spin $\boldsymbol{I}$ in an external magnetic field $\boldsymbol{H}_{0}$ coupled to the surrounding electron spins $\boldsymbol{S}_{i}$ is

$$
H_{N}=\gamma_{N} \boldsymbol{H}_{0} \cdot \boldsymbol{I}+A_{\mathrm{hf}} \sum_{i} \boldsymbol{S}_{i} \cdot \boldsymbol{I},
$$

where $\gamma_{N}$ denotes the nuclear gyromagnetic ratio and $A_{\mathrm{hf}}$ is the coupling constant of the isotropic (supertransferred) hyperfine interaction. In the presence of a nonmagnetic impurity the local spin susceptibility that determines the on-site spin polarization is altered according to Eq. (28), yielding for the impurity contribution to the Knight shift at distance $\boldsymbol{R}$ from the vacancy

$$
\delta K(T ; \boldsymbol{R})=\frac{\gamma A_{\mathrm{hf}}}{\gamma_{N}} \sum_{i} \delta \chi\left(T ; \boldsymbol{R}+\boldsymbol{r}_{i}\right),
$$

where the sum is over nearest-neighbor lattice sites of the nucleus being probed and $\gamma=g \mu_{B}$ with the $g$ factor of the $\mathrm{Cu}^{2+}$ spin and the Bohr magneton $\mu_{B}$. Taking into account only coupling to the most dominant moments that reside on the nearest-neighbor sites surrounding the vacancy, Eq. (35) becomes

$$
\delta K(T)=\frac{\gamma A_{\mathrm{hf}}}{\gamma_{N}} \frac{n}{2 \pi T} f(T) \ln ^{-1} \frac{D}{T} .
$$

The correction factor 


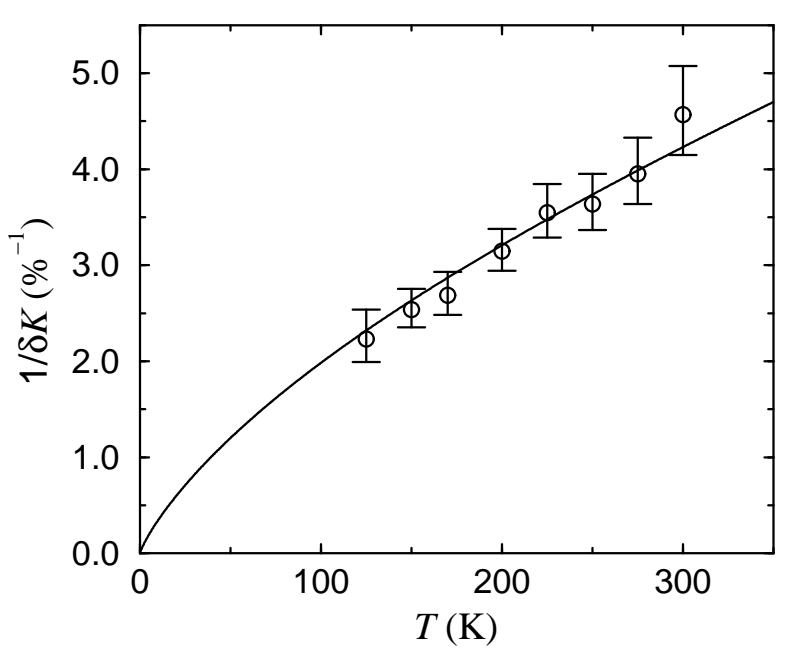

FIG. 1. Temperature-dependence of the inverse of the impurity-induced Knight shift $1 / \delta K(T)$ on the impurity nucleus. The solid line is a fit employing Eq. (36) to experimental data by Ishida et al. for $3 \%{ }^{27} \mathrm{Al}$-doped $\mathrm{La}_{1.85} \mathrm{Sr}_{0.15} \mathrm{CuO}_{4}$ indicated by circles.

$$
f(T)=\left(y K_{1}(y)\right)^{2}, \quad y=\frac{4 \sqrt{2} T}{\pi D},
$$

reduces to unity in the limit $T \ll J$. The variable $n$ in Eq. (36) denotes the number of sites that are nearest neighbors to the vacancy as well as to the nucleus being probed.

The Knight shift on the impurity nucleus is influenced predominantly by the local moments residing on the four nearest-neighbor sites of the impurity as described by Eq. (36) with $n=4$. Figure 11 contains the calculated $T$ dependence of $1 / \delta K(T)$. We set $J=1500 \mathrm{~K}$ and $g=2$; by varying the supertransferred hyperfine coupling constant $A_{\mathrm{hf}}$ the curve is fitted to experimental data obtained by Ishida et al. 6 for the Knight shift of ${ }^{27} \mathrm{Al}$ performed on $3 \%{ }^{27} \mathrm{Al}$-doped $\mathrm{La}_{1.85} \mathrm{Sr}_{0.15} \mathrm{CuO}_{4}$. From the fit we find for the supertransferred hyperfine coupling constant a numerical value of ${ }^{27} A_{\mathrm{hf}} / \gamma_{\text {scriptsize } \mathrm{N}}=18.2 \mathrm{k}$ Gs. This compares well with ${ }^{27} A_{\mathrm{hf}} / \gamma_{N}=16 \mathrm{k}$ Gs found by Ishida et al.

Upon doping the system with impurities, the NMR signal of $\mathrm{Cu}$ and $\mathrm{Y}$ is observed to split into a main line accompanied by satellite line(s) as is discussed in the following. Within the approximation employed in Eq. (36) we assume the impurity-induced moments to reside predominantly on the nearest-neighbor sites of the vacancy. Resonance signals originating from nuclei that do not lie in the vicinity of the impurity are affected only little by these moments and contribute to the NMR main line. Resonance signals from $\mathrm{Cu}$ nuclei on nearest-neighbor sites of the impurity are shifted with respect to the main line as described by Eq. (36) with $n=1$ and constitute a single $\mathrm{Cu}$ satellite line. Resonance signals from Y nuclei located on nearest-neighbor and next-nearest neighbor sites of the impurity are seen as two satellite lines corresponding to $n=2$ and $n=1$, respectively. These $\mathrm{Y}$ satellite lines have been observed experimentally by Mahajan et al. 1 in $1 \% \mathrm{Zn}$-doped $\mathrm{YBa}_{2} \mathrm{Cu}_{3} \mathrm{O}_{6.64}$.

We now discuss briefly the NMR line-broadening which results from the inhomogeneous distribution of local Knight shifts in the presence of many vacancies. According to Eq. (28) moments induced by a vacancy located on the $A$ sublattice are found on all sites of sublattice $B$ with magnitude $\propto R^{-2}, R$ being the distance from the vacancy. As a result the nuclear resonance signals contributing to the main and satellite lines vary with distance from the impurities which can be observed as a broadening of the line. At a finite concentration $c$ of vacancies the linewidth $\Delta H$ can be estimated from Eq. (35), assuming an average distance between vacancies of $R_{\mathrm{av}}=\sqrt{1 / c}$ :

$$
\frac{\Delta H}{H_{0}} \approx \frac{\gamma A_{\mathrm{hf}}}{\gamma_{N}} \frac{c}{2 \pi T} \ln ^{-1} \frac{D}{T} .
$$

\section{IMPURITY INTERACTION}

In this section we study the interaction between two vacancies, first by considering two isolated impurities. The case of a finite concentration of impurities is treated subsequently.

The spinon Green's function in the presence of two impurities located at sites 0 and $\boldsymbol{R}$ is

$$
G_{\boldsymbol{R}}\left(i \omega_{n}\right)=N G^{0}\left(i \omega_{n}\right)+\delta G_{\boldsymbol{R}}\left(i \omega_{n}\right) .
$$

Employing scattering theory the two-impurity correction $\delta G_{\boldsymbol{R}}\left(i \omega_{n}\right)$ can be expressed as

$$
\begin{aligned}
& \delta G_{\boldsymbol{R}}\left(i \omega_{n}\right)= \\
& \frac{1}{N} \sum_{\boldsymbol{r}}\left(\begin{array}{c}
G^{0}\left(i \omega_{n} ; \boldsymbol{r}, 0\right) \\
G^{0}\left(i \omega_{n} ; \boldsymbol{r}, \boldsymbol{R}\right)
\end{array}\right) \boldsymbol{T}_{\boldsymbol{R}}\left(i \omega_{n}\right)\left(\begin{array}{c}
G^{0}\left(i \omega_{n} ; 0, \boldsymbol{r}\right) \\
G^{0}\left(i \omega_{n} ; \boldsymbol{R}, \boldsymbol{r}\right)
\end{array}\right)
\end{aligned}
$$

with the unperturbed Green's function $G^{0}\left(i \omega_{n} ; \boldsymbol{R}, \boldsymbol{R}^{\prime}\right)=$ $-\left\langle T_{\tau} f_{\boldsymbol{R}}(\tau) f_{\boldsymbol{R}^{\prime}}^{\dagger}(0)\right\rangle_{\omega_{n}}^{0}$ and the scattering matrix

$$
\boldsymbol{T}_{\boldsymbol{R}}\left(i \omega_{n}\right)=\left(\begin{array}{ll}
T_{11} & T_{12} \\
T_{21} & T_{22}
\end{array}\right)
$$

with

$$
\begin{aligned}
& T_{11}=T_{22}=-1 / G\left(i \omega_{n}, \boldsymbol{R}, \boldsymbol{R}\right), \\
& T_{12}=G^{0}\left(i \omega_{n} ; 0, \boldsymbol{R}\right) /\left[G\left(i \omega_{n} ; \boldsymbol{R}, \boldsymbol{R}\right) G^{0}\left(i \omega_{n}\right)\right], \\
& T_{21}=G^{0}\left(i \omega_{n} ; \boldsymbol{R}, 0\right) /\left[G\left(i \omega_{n} ; \boldsymbol{R}, \boldsymbol{R}\right) G^{0}\left(i \omega_{n}\right)\right] .
\end{aligned}
$$

The diagonal and off-diagonal elements of $\boldsymbol{T}_{\boldsymbol{R}}\left(i \omega_{n}\right)$ describe successive scattering by one impurity and by the two impurities, respectively. From Eqs. (40)-(42) one obtains 


$$
\delta G_{\boldsymbol{R}}\left(i \omega_{n}\right)=\frac{\partial}{\partial i \omega_{n}} \ln \left(G^{0}\left(i \omega_{n}\right) G\left(i \omega_{n} ; \boldsymbol{R}, \boldsymbol{R}\right)\right),
$$

where $G\left(i \omega_{n} ; \boldsymbol{R}, \boldsymbol{R}\right)$ is given by Eq. (21).

By means of Eq. (43) we evaluate the two-impurity contribution to the density of states $\delta \rho_{\boldsymbol{R}}(\omega)=$ $-1 / \pi \operatorname{Im} \delta G_{\boldsymbol{R}}\left(\omega+0^{+}\right)$. For $\boldsymbol{R} \in B$ one finds a splitting of the resonance energy level

$$
\delta \rho_{\boldsymbol{R} \in B}(\omega)=\delta(\omega-J(\boldsymbol{R}))+\delta(\omega+J(\boldsymbol{R})),
$$

where the parameter $J(\boldsymbol{R})$ controlling the level splitting is given by

$$
J(\boldsymbol{R})=\frac{D \sqrt{2 \Phi_{B}(\boldsymbol{R})}}{2 R} \ln ^{-1} \frac{\pi R}{\sqrt{2 \Phi_{B}(\boldsymbol{R})}} .
$$

The level splitting results in an attraction between the two impurities due to the formation of a singlet of the induced moments. For the shortest distance between vacancies, $R=1$, Eq. (45) gives $J(\boldsymbol{R}) \approx J$ as expected: $J$ is the only energy scale of the model under consideration. In the present mean-field theory no attraction is found between impurities lying on different sublattices, i.e., $\boldsymbol{R} \in A$. These findings are also consistent with exact diagonalization data of Bulut et al. 18 who found a binding energy of $-0.56 J$ for nearest-neighbor static vacancies on a $4 \times 4$ lattice, while the interaction between vacancies separated by $\boldsymbol{R}$ is negligible if $R_{x}+R_{y}$ is an even number. We interprete $J(\boldsymbol{R})$ given by Eq. (45) as being the antiferromagnetic exchange coupling of impurity moments. Indeed, the impurity contribution to the spin susceptibility calculated from Eq. (43) is

$$
\begin{aligned}
& \delta \chi(T \gg J(\boldsymbol{R}))=\frac{1}{2 T}, \\
& \delta \chi(T \ll J(\boldsymbol{R}))=\frac{2}{T} \exp \left(-\frac{J(\boldsymbol{R})}{T}\right),
\end{aligned}
$$

which is precisely that of two $1 / 2$ spins coupled by an interaction $H_{\text {int }}=J(\boldsymbol{R}) \boldsymbol{S}_{1} \cdot \boldsymbol{S}_{2}$.

From Eq. (45) the interaction potential between two moments is found to fall off slowly as $R^{-1}$ with distance between the vacancies. In a real system with finite impurity concentration $c$, however, one expects this interaction to be screened at large distance by the presence of other impurities. To account for this effect we introduce a finite self-energy $\Sigma(\omega)$ determined by means of a coherent-potential approximation $(\mathrm{CPA})$ :

$$
\Sigma(\omega) G^{0}(\omega-\Sigma(\omega))+c=0 .
$$

Neglecting the frequency dependence of $\Sigma(\omega)$ we approximate the self-energy by $\Sigma(\omega) \approx \Sigma(\omega \rightarrow 0)=-i\left|\Sigma_{0}^{\prime \prime}\right|$, where $\Sigma_{0}^{\prime \prime}$ fulfills

$$
-\frac{4}{\pi D^{2}} \Sigma_{0}^{\prime \prime 2} \ln \frac{D}{\left|\Sigma_{0}^{\prime \prime}\right|}+c=0
$$

Reevaluating the exchange coupling parameter $J(\boldsymbol{R})$ one finds an exponential cutoff in the two-impurity interaction potential

$$
J(\boldsymbol{R}) \propto \exp \left(-R / R_{\mathrm{av}}\right),
$$

where $R_{\mathrm{av}}=\sqrt{1 / c}$ is the average distance between impurities. For small distances $R \ll J$ one recovers again Eq. (45). The present CPA treatment gives a qualitative description of the screening of the interaction potential of two distant impurities, but it neglects important effects such as the formation of spin singlets among closely spaced moments; a complete analysis of the manyimpurity problem should take this effect into account.

Having the interaction scale between moments we can give an estimation of the relaxation rate $1 / T_{1}$ of nuclear magnetization resulting from fluctuations of the impurity-induced moments in the vicinity of the vacancy. To be specific let us consider the nuclear-spin relaxation rate $1 / T_{1}$ at the impurity site (obtained, for instance, from the ${ }^{27} \mathrm{Al}-\mathrm{NMR}$ signal in an experiment by Ishida et al. 6 ) With the supertransferred hyperfine interaction of Eq. (34) the nuclear relaxation rate is

$$
\frac{1}{T_{1}} \approx \frac{2}{3} \tau A_{\mathrm{hf}}^{2} \sum_{\boldsymbol{\delta} \boldsymbol{\delta}^{\prime}}\left\langle\boldsymbol{S}_{\boldsymbol{\delta}} \cdot \boldsymbol{S}_{\boldsymbol{\delta}^{\prime}}\right\rangle,
$$

where $\tau$ is the correlation time of the local moment and $\hbar=1$. The amplitude of spin fluctuations on nearestneighbor sites of the impurity is given by the equal-time spin-correlation function

$$
\begin{aligned}
& \sum_{\delta \boldsymbol{\delta}^{\prime}}\left\langle\boldsymbol{S}_{\boldsymbol{\delta}} \cdot \boldsymbol{S}_{\boldsymbol{\delta}^{\prime}}\right\rangle \\
& =-3 T^{2} \sum_{i \omega_{n}, i \nu_{m}} \sum_{\boldsymbol{\delta} \boldsymbol{\delta}^{\prime}} \delta G\left(i \omega_{n}+i \nu_{m} ; \boldsymbol{\delta}, \boldsymbol{\delta}^{\prime}\right) \delta G\left(i \omega_{n} ; \boldsymbol{\delta}^{\prime}, \boldsymbol{\delta}\right) \\
& =3\left(\frac{z}{\pi}\right)^{2} \ln ^{-2} \frac{D}{T}
\end{aligned}
$$

where $\delta G\left(i \omega_{n} ; \boldsymbol{\delta}, \boldsymbol{\delta}^{\prime}\right)=-B^{*}\left(i \omega_{n} ; \boldsymbol{\delta}\right) B\left(i \omega_{n} ; \boldsymbol{\delta}^{\prime}\right) / G^{0}\left(i \omega_{n}\right)$, $\nu_{m}=2 m \pi T$ are bosonic frequencies, and $z=4$ is the number of nearest neighbors. We assume the correlation time $\tau$ in Eq. (50) to be dominated by the exchange interaction among moments. In principle, $\tau$ has a certain distribution due to the random spreading of impurities. We do not go into this delicate issue and roughly approximate $\tau \approx 1 /\left[z J\left(\sqrt{2} R_{\text {av }}\right)\right]$, where $J\left(\sqrt{2} R_{\text {av }}\right)$ is given by Eq. (45) and $\sqrt{2} R_{\mathrm{av}}=\sqrt{2 / \mathrm{c}}$ is the average distance between impurities located on different sublattices. One then obtains

$$
\tau \approx \frac{\sqrt{2}}{z D \sqrt{c}} \ln \frac{2 \pi^{2}}{c} .
$$

Assuming an impurity concentration $c=3 \%$, a temperature $T=100 \mathrm{~K}$, and using the value of the supertransferred hyperfine coupling constant $A_{\mathrm{hf}}$ found in the previous section, Eqs. (50)-(52) give $1 / T_{1} \approx 0.29 \mathrm{~m} \mathrm{~s}^{-1}$. We compare this value to $1 / T_{1}=0.43 \mathrm{~m} \mathrm{~s}^{-1}$ observed by Ishida et al. 5 for $3 \%{ }^{27} \mathrm{Al}$-doped $\mathrm{La}_{1.85} \mathrm{Sr}_{0.15} \mathrm{CuO}_{4}$ and find it to be of the same order of magnitude. 


\section{KONDO SCREENING OF LOCAL MOMENTS}

The two-dimensional Heisenberg model has a Néelordered ground state and it was implicitly assumed above that the spin-gap disordered state is stabilized by mobile holes. Naturally, the question then arises how a local moment induced by a static vacancy will be affected by these charge carriers. Once the metallic state with finite Fermi surface is formed one usually expects Kondo screening of the local moment at low temperature. As an exception, a spinon bound state on a vacancy can coexist with metallic conductivity if spin-charge separation occurs with a gap in the spin-excitation spectrum. Underdoped cuprates, however, seem to have ungapped pieces of their original (large) Fermi surfaces even in a "spin-gap" phase19. Therefore the bound spinon on the vacancy is screened by gapless Fermi-surface excitations, although the Kondo temperature is expected to be low due to the reduced density of states. The characteristic Kondo-energy scale can be roughly estimated in the following way.

We introduce fermionic field operators $\psi_{i \sigma}=s_{\sigma} \varphi\left(\boldsymbol{R}_{i}\right)$ which annihilate a spinon $s_{\sigma}$ in a bound state with wave function $\varphi\left(\boldsymbol{R}_{i}\right), \boldsymbol{R}_{i}$ being the distance from the spinless vacancy. Due to the hopping term in the $t-J$ model there is a finite amplitude $t \varphi\left(\boldsymbol{R}_{i}\right)$ of mixing of the bound spinon with extended states:

$$
H=t \sum_{i, \boldsymbol{\delta}, \sigma}\left(c_{i+\boldsymbol{\delta}, \sigma}^{\dagger} h_{i}^{\dagger} \psi_{i, \sigma}+\text { H.c. }\right) \text {. }
$$

The bosonic operators $h_{i}^{\dagger}$ and $c_{i+\boldsymbol{\delta}, \sigma}^{\dagger}$ in Eq. (53) create a "holon" and a conduction electron, respectively. In a slave-boson formulation, the latter is a product of the lattice spinon and holon operators.

At finite doping, $x=\left\langle\left|h_{i}\right|^{2}\right\rangle \neq 0$, the Hamiltonian (53) allows for a localized spinon to escape the impurity which leads to a broadening of the bound state. The width of this resonance controls the low-temperature behavior of the local spin and is given by the $s_{\sigma}$ fermion self-energy

$$
\begin{aligned}
\Sigma^{s}(\omega)= & t^{2} T \sum_{\nu} \sum_{i j, \boldsymbol{\delta} \boldsymbol{\delta}^{\prime}} \varphi^{*}\left(\boldsymbol{R}_{i}\right) \varphi\left(\boldsymbol{R}_{j}\right) G_{\nu}^{h}\left(\boldsymbol{R}_{i j}\right) \\
& \times G_{\omega-\nu}^{c}\left(\boldsymbol{R}_{i+\boldsymbol{\delta}}-\boldsymbol{R}_{j+\boldsymbol{\delta}^{\prime}}\right) .
\end{aligned}
$$

At low temperatures the holons are almost condensed, and the holon Green's function can be approximated by $G_{\nu}^{h} \approx(x / T) \delta_{\nu, 0}$. Furthermore, we keep only the nonoscillatory on-site terms that present the leading contributions to Eq. (54) and employ the normalization condition $\sum_{i}\left|\varphi_{i}\right|^{2}=1$. Then the width of the Kondo resonance is

$$
\operatorname{Im} \Sigma^{s}(0) \approx \pi z x t^{2} N^{c}(0)
$$

where $N^{c}(0)=1 / \pi \sum_{k} \operatorname{Im} G_{k}^{c}(\omega=0)$ is the on-site density of electronic states on the Fermi level.

In the overdoped $t-J$ model with a large gapless Fermi surface the density of states is controlled by the inverse of the full bandwidth, $N^{c}(0) \approx 1 /(2 z t)$. In the underdoped pseudogap regime, $x t \ll J$, the density of states $N^{c}(0)$ is strongly reduced which is due to the fact that the ungapped areas of the Fermi surface contributing to $N^{c}(0)$ are only small. We simply assume $N^{c}(0) \approx x /(2 z t)$ and estimate the Kondo temperature using Eq. (55) as

$$
T_{K}(x) \approx \frac{\pi}{2} x^{2} t
$$

For a typical value of $x \approx 0.1$ and $t \approx 0.4 \mathrm{eV}$ this gives $T_{K} \approx 70 \mathrm{~K} . T_{K}(x)$ quickly increases with $x$, causing the vacancy-induced moment to disappear in the optimal and overdoped regime. This magnetic-nonmagnetic Kondo crossover upqn hole doping was recently employed by Nagaosa and Lee 2 to explain the unusual doping dependence of the residual resistivity of cuprate superconductors.

One may wonder why the exchange parameter $J$ does not enter in Eq. (56). In fact, the main role of the Heisenberg term in this quite unusual Kondo-like behavior of a spinless vacancy is to produce a spin pseudogap hence creating a local moment. It is the hopping term that is then responsible for converting the spinon bound state into a resonance of finite width.

\section{CONCLUSION}

In summary, we have studied the localized magnetic states induced by static spin vacancies in underdoped high- $T_{c}$ cuprates. Starting from the two-dimensional Heisenberg antiferromagnet we employ a drone-fermion mean-field theory describing a spin liquid in the spingap phase. The impurity induces a spinon bound state residing predominantly on the nearest-neighbor sites of the impurity. We have calculated the impurity-induced Knight shift and found it in ggod agreement with experimental data of Ishida et al. 6 for Al-doped cuprates. The calculations do also account for the appearance of satellite peaks in NMR measurements on Y observed by Mahajan et al.5 in Zn-doped cuprates. Two impurities are found to attract each other due to the formation of a singlet of the induced moments. In the presence of a finite concentration of impurities the interaction between moments is screened at distances larger then the average separation between impurities. We estimate the contribution of local moments to the NMR relaxation rate which is found to be in reasonable agreement with experiment. Finally, an estimation of the Kondo temperature of screening of the vacancy-induced moments by charge carriers is given, below which a nonmagnetic impurity behaves as a sharp resonance at the Fermi level. 
${ }^{1}$ G. Xiao et al., Phys. Rev. B 35, 8782 (1987).

${ }^{2}$ A. M. Finkelstein, V. E. Kataev, E. F. Kukovitskii, and G. B. Teitelbaum, Physica C 168, 370 (1990).

${ }^{3}$ H. Alloul et al., Phys. Rev. Lett. 67, 3140 (1991).

${ }^{4}$ G. Zheng et al., J. Phys. Soc. Jpn. 62, 2591 (1993).

${ }^{5}$ A. V. Mahajan, H. Alloul, G. Collin, and J. F. Marucco, Phys. Rev. Lett. 72, 3100 (1994).

${ }^{6}$ K. Ishida et al., Phys. Rev. Lett. 76, 531 (1996).

${ }^{7}$ M. Sigrist and A. Furusaki, J. Phys. Soc. Jpn. 65, 2385 (1996).

${ }^{8}$ N. Nagaosa, A. Furusaki, M. Sigrist, and H. Fukuyama, J. Phys. Soc. Jpn. 65, 3724 (1996).

${ }^{9}$ N. Nagaosa and T.-K. Ng, Phys. Rev. B 51, 15588 (1995).

${ }^{10}$ S. Krivenko and G. Khaliullin, JETP Lett. 62, 723 (1995).

${ }^{11}$ P. W. Anderson, Science 235, 1196 (1987).

${ }^{12}$ I. Affleck, T. Kennedy, E. H. Lieb, and H. Tasaki, Phys. Rev. Lett. 59, 799 (1987).

${ }^{13}$ D. C. Mattis, The Theory of Magnetism I (Springer-Verlag, Berlin, 1981), p. 90.

${ }^{14}$ I. Affleck and J. B. Marston, Phys. Rev. B 37, 3774 (1988).

${ }^{15}$ G. Kotliar, Phys. Rev. B 37, 3664 (1988).

${ }^{16}$ S. Krivenko and G. Khaliullin, Physica C 244, 83 (1995); see also A. M. Tsvelik, Phys. Rev. Lett. 69, 2142 (1992), where the real (Majorana) fermion based treatment of quantum disordered spin systems was suggested.

${ }^{17}$ I. S. Gradshteyn and I. M. Ryzhik, in Table of Integrals, Series and Products, 5th ed. edited by A. Jeffrey (Academic, Boston, 1994), Chap. 8.

${ }^{18}$ N. Bulut, D. Hone, D. J. Scalapino, and E. Y. Loh, Phys. Rev. Lett. 62, 2192 (1989). Note that our definition of the exchange parameter $J$ differs from that of Bulut et al. by a factor of 2 .

19 A. G. Loeser et al., Science 273, 325 (1996); H. Ding et al., Nature (London) 382, 51 (1996).

${ }^{20}$ N. Nagaosa and P.A. Lee, cond-mat/9702103 (unpublished). 